\title{
The gluon propagator in Feynman gauge by the method of stationary variance
}

\author{
Fabio Siringo \\ Dipartimento di Fisica e Astronomia dell'Università di Catania, \\ INFN Sezione di Catania, Via S.Sofia 64, I-95123 Catania, Italy
}

(Dated: July 6, 2018)

\begin{abstract}
The low-energy limit of pure Yang-Mills $S U(3)$ gauge theory is studied in Feynman gauge by the method of stationary variance, a genuine second-order variational method that is suited to deal with the minimal coupling of fermions in gauge theories. In terms of standard irreducible graphs, the stationary equations are written as a set of coupled non-linear integral equations for the gluon and ghost propagators. A physically sensible solution is found for any strength of the coupling. The gluon propagator is finite in the infrared, with a dynamical mass that decreases as a power at high energies. At variance with some recent findings in Feynman gauge, the ghost dressing function does not vanish in the infrared limit and a decoupling scenario emerges as recently reported for the Landau gauge.

PACS numbers: 12.38.Lg,12.38.Aw,14.70.Dj,11.15.Tk
\end{abstract}

\section{INTRODUCTION}

There is a growing consensus on the utility of variational methods as analytical tools for a deeper understanding of the infrared (IR) limit of non-Abelian gauge theories. The IR slavery of these theories makes the standard perturbation theory useless below some energy scale, and our theoretical knowledge of the IR limit relies on lattice simulation and on non-perturbative techniques like functional renormalization group [1] and Dyson-Schwinger equations[2]. Variational methods have been developed as a complement to these analytical approaches, and their utility has been proven by several authors in the last years 3 9|. Quite recently, the method of stationary variance 10, 11] has been advocated as a powerful second order extension of the Gaaussian Effective Potential (GEP) 12 15]. The GEP is a genuine variational method and has been successfully applied to many physical problems in field theory, from scalar and electroweak theories 15-22 to superconductivity 23-25] and antiferromagnetism[26], but turns out to be useless for gauge interacting fermions[27]. Actually, since the GEP only contains first order terms, it is not suited for describing the minimal coupling of gauge theories that has no first-order effects. Several methods have been explored for including fermions 22] and higher order corrections 28], sometimes spoiling the genuine variational character of the method.

By a formal higher order extension of the GEP 29] the method of stationary variance has been developed as a genuine variational method that keeps in due account second order effects and seems to be suited to deal with the minimal coupling of gauge theories. While the method has been shown to be viable for the simple Abelian case of QED [30], its full potentialities have not been explored yet. As a non-perturbative tool that can deal with fermions in gauge theories, the method seems to be very useful for exploring the IR limit of QCD, and its natural application field is the non-Abelian $S U(3)$ gauge theory.
While a full study of QCD by that method is still far away, as a first step, in this paper we explore the solution of the stationary equations for pure Yang-Mills $S U(3)$ theory. The method of stationary variance provides a set of non-linear coupled integral equations whose solutions are the propagators for gluons and ghosts. Therefore the work has a double motivation: the technical aim of showing that the method is viable and a solution does exist (which is not obvious nor proven in general), and the physical interest on the gluon propagator in the IR limit, where its properties seem to be related to the important issue of confinement.

On the technical side, having shown that a sensible untrivial solution does exist is a major achievement that opens the way to a broader study of QCD by the same method. Inclusion of quarks would be straightforward as some fermions, the ghosts, are already present in the simple Yang-Mills theory, and they seem to play well their role of canceling the unphysical degrees of freedom. Other important technical issues are gauge invariance, renormalization and the choice of a physical scale.

The method is not gauge invariant, and we did not make any effort to restore gauge invariance at this stage. There are several ways to attempt it 3 , 31], but in this first step we preferred to fix a gauge, namely the Feynman gauge where the calculation is easier, and explore the properties of the solution to see if any unphysical feature emerges for the propagator and the polarization function. Actually the polarization function is found approximately transverse up to a constant mass shift due to the dynamical mass generation. As far as the solution satisfies, even approximately, the constraints imposed by gauge invariance, the method is acceptable on the physical ground. On the other hand the gluon propagator is not a physical observable and is known to be a gaugedependent quantity. Of course, since the solution depends on the gauge, the choice of working in Feynman gauge could be non-optimal, and the method could be improved by exploring other gauge choices, like Landau gauge. Besides being easier, working in Feynman gauge is 
also interesting from the physical point of view, as there are very few data available on the gluon propagator in this gauge.

Since lattice simulations are the most natural benchmark for any variational calculation in the IR limit, we borrowed from lattice simulation the regulating scheme in terms of an energy cutoff and a bare coupling that depends on it. Renormalization Group (RG) invariance requires that the physical observables are left invariant by a change of the cutoff that is followed by the corresponding change of the bare coupling. Then, renormalized physical quantities can be defined that do not depend on the cutoff. The only free parameter of the theory is the energy scale, that must be fixed by a comparison with the experimental data or lattice simulations. No other fit parameter has been introduced in the method. Moreover, we do not need to insert any counterterm and especially mass counterterms that are forbidden by the gauge invariance of the Lagrangian.

On the physical side, the properties of the gluon propagator in Feynman gauge are basically unexplored. In Coulomb gauge [4 7, , 9] and in Landau gauge [8, 31 39] there has been an intense theoretical work in the last years. In Landau gauge theoretical and lattice data are generally explained in terms of a decoupling regime, with a finite ghost dressing function and a finite massive gluon propagator. The more recent findings confirm the prediction of a dynamical mass generation for the gluon[40]. In Feynman gauge we do not expect a very different scenario. A finite ghost propagator has been recently proposed[41], but there are no lattice data available that could confirm it. That makes the study of the Feynman gauge more interesting. In the present work no important differences are found with respect to the Landau gauge. A decoupling scenario emerges, with very flat ghost dressing functions, flatter than expected, and a finite gluon propagator in the IR limit. A dynamical mass is found that saturates at about $0.8 \mathrm{GeV}$ and decreases as a power in the high energy limit. Unfortunately the quantitative predictions are biased by an approximate estimate of the energy scale due to the lack of lattice data in Feynman gauge.

This paper is organized as follows: in Section II the method is described in detail for the special case of pure $S U(3)$ Yang-Mills theory; then in Section III the stationary equations are derived and written in terms of standard irreducible Feynman graphs; a comparison with other recent variational approaches is reported in Section IV where some aspects of the method are clarified; in Section $\mathrm{V}$ the renormalization scheme is discussed and the numerical solutions are studied in great detail, comparing them with the available lattice data; in Section V a second-order approximation is introduced, and the numerical solution is proposed as a better approximation for the propagator; finally, in Section VI the results are discussed and several lines for future work are outlined. Details on the numerical calculation and explicit integral expressions for the Feynman graphs are reported in the appendix.

\section{SETUP OF THE METHOD}

The method of stationary variance [10, 11] is a second order variational technique that is suited to describe gauge theories with a minimal coupling like gauge theories [29, 30], where first order approximations like the GEP do not add anything to the standard treatment of perturbation theory 27|. The method has been discussed in some detail in Ref.[29] and applied to QED in Ref.[30]. Here we give the main details for a non-Abelian theory like $S U(3)$.

Let us consider a pure Yang-Mills $S U(3)$ gauge theory without external fermions. The Lagrangian can be written as

$$
\mathcal{L}=\mathcal{L}_{Y M}+\mathcal{L}_{f i x}
$$

where $\mathcal{L}_{Y M}$ is the Yang-Mills term

$$
\mathcal{L}_{Y M}=-\frac{1}{2} \operatorname{Tr}\left(\hat{F}_{\mu \nu} \hat{F}^{\mu \nu}\right)
$$

and $\mathcal{L}_{f i x}$ is a guage fixing term. In terms of the gauge fields, the tensor operator $\hat{F}_{\mu \nu}$ reads

$$
\hat{F}_{\mu \nu}=\partial_{\mu} \hat{A}_{\nu}-\partial_{\nu} \hat{A}_{\mu}-i g\left[\hat{A}_{\mu}, \hat{A}_{\nu}\right]
$$

where

$$
\hat{A}_{\mu}=\sum_{a} \hat{X}^{a} A_{\mu}^{a}
$$

and the generators of $S U(3)$ satisfy the algebra

$$
\left[\hat{X}^{a}, \hat{X}^{b}\right]=i f_{a b c} \hat{X}^{c}
$$

with the structure constants normalized according to

$$
f_{a b c} f_{d b c}=N \delta_{a d}
$$

and $N=3$. Quite generally, the gauge-fixing term can be taken as

$$
\mathcal{L}_{f i x}=-\frac{1}{\xi} \operatorname{Tr}\left[\left(\partial_{\mu} \hat{A}^{\mu}\right)\left(\partial_{\nu} \hat{A}^{\nu}\right)\right]
$$

and the quantum effective action $\Gamma\left[A^{\prime}\right]$, as a function of the external background field $A^{\prime}$ can be written

$$
e^{i \Gamma\left[A^{\prime}\right]}=\int_{1 P I} \mathcal{D}_{A} e^{i S\left[A^{\prime}+A\right]} J_{F P}\left[A^{\prime}+A\right]
$$

where $S[A]$ is the action, $J_{F P}[A]$ is the Faddev-Popov determinant and the path integral represents a sum over one particle irreducible (1PI) graphs 42]. Since the gauge symmetry is not broken and we are mainly interested in the propagators, in the present paper we will limit to the 
physical vacuum at $A^{\prime}=0$, while a more general formalism can be developed for a full study of the vertex functions by keeping $A^{\prime} \neq 0$ in order to take the derivatives of the effective action[18].

The determinant $J_{F P}$ can be expressed as a path integral over ghost fields

$$
J_{F P}[A]=\int \mathcal{D}_{\omega, \omega \star} e^{i S_{g h}\left[A, \omega, \omega^{\star}\right]}
$$

and the effective action can be written as

$$
e^{i \Gamma}=\int_{1 P I} \mathcal{D}_{A, \omega, \omega^{\star}} e^{i S_{0}\left[A, \omega, \omega^{\star}\right]} e^{i S_{I}\left[A, \omega, \omega^{\star}\right]}
$$

where of course, the total action is

$$
S_{\text {tot }}=S_{0}+S_{I}=\int \mathcal{L}_{Y M} \mathrm{~d}^{4} x+\int \mathcal{L}_{f i x} \mathrm{~d}^{4} x+S_{g h}
$$

but we have the freedom to split it in the two parts, the free action $S_{0}$ and the interaction $S_{I}$, by insertion of trial functions [29]. We define the free action $S_{0}$ as

$$
\begin{aligned}
S_{0} & =\frac{1}{2} \int A^{a \mu}(x) D_{\mu \nu}^{-1}(x, y) A^{b \nu}(y) \mathrm{d}^{4} x \mathrm{~d}^{4} y \\
& +\int \omega_{a}^{\star}(x) G^{-1}{ }_{a b}(x, y) \omega_{b}(y) \mathrm{d}^{4} x \mathrm{~d}^{4} y
\end{aligned}
$$

where $D_{\mu \nu}^{a b}(x, y)$ and $G_{a b}(x, y)$ are unknown trial matrix functions. The interaction then follows by difference

$$
S_{I}=S_{t o t}-S_{0}
$$

and can be formally written as the sum of a two-point term and three local terms: the ghost vertex, the threegluon vertex and the four-gluon vertex respectively

$$
S_{I}=S_{2}+\int \mathrm{d}^{4} x\left[\mathcal{L}_{g h}+\mathcal{L}_{3}+\mathcal{L}_{4}\right]
$$

In detail, the two-point interaction term can be written as

$$
\left.S_{2}=\frac{1}{2} \int A^{a \mu}(x)\left[D_{0}^{-1^{a b}}(x, y)-D_{\mu \nu}^{-1} a b, y\right)\right] A^{b \nu}(y) \mathrm{d}^{4} x \mathrm{~d}^{4} y+\int \omega_{a}^{\star}(x)\left[G_{0}^{-1}{ }_{a b}(x, y)-G_{a b}^{-1}(x, y)\right] \omega_{b}(y) \mathrm{d}^{4} x \mathrm{~d}^{4} y
$$

where $D_{0}$ and $G_{0}$ are the standard free-particle propagators for gluons and ghosts and their Fourier transforms read

$$
\begin{aligned}
D_{0 \mu \nu}^{a b}(p) & =-\frac{\delta_{a b}}{p^{2}}\left[\eta_{\mu \nu}+(\xi-1) \frac{p_{\mu} p_{\nu}}{p^{2}}\right] \\
G_{0 a b}(p) & =\frac{\delta_{a b}}{p^{2}}
\end{aligned}
$$

where $\eta_{\mu \nu}$ is the metric tensor. The three local interaction terms are

$$
\begin{aligned}
\mathcal{L}_{3} & =-g f_{a b c}\left(\partial_{\mu} A_{\nu}^{a}\right) A^{b \mu} A^{c \nu} \\
\mathcal{L}_{4} & =-\frac{1}{4} g^{2} f_{a b c} f_{a d e} A_{\mu}^{b} A_{\nu}^{c} A^{d \mu} A^{e \nu} \\
\mathcal{L}_{g h} & =-g f_{a b c}\left(\partial_{\mu} \omega_{a}^{\star}\right) \omega_{b} A^{c \mu} .
\end{aligned}
$$

The trial functions $G_{a b}, D_{\mu \nu}^{a b}$ cancel in the total action $S_{\text {tot }}$ which is exact and cannot depend on them. Thus this formal decomposition holds for any arbitrary choice of the trial functions, provided that the integrals converge. Standard Feynman graphs can be drawn for this theory with the trial propagators $D_{\mu \nu}^{a b}$ and $G_{a b}$ that play the role of free propagators, and the vertices that can be read from the interaction action $S_{I}$ in Eq.(14). As shown in Fig.1, we have two-particle vertices for gluons and ghosts that arise from the action term $S_{2}$ in Eq.(15), while the local terms in Eq. (17) give rise to three- and four-particle vertices.
While the effective action $\Gamma$ can be evaluated by perturbation theory order by order, as a sum of Feynman diagrams, a genuine variational method can be established by the functional derivative of the effective potential with respect to the trial propagators, in order to fulfill some given stationary conditions. However, as recently discussed 29], the stationary conditions can be written in terms of self-energy graphs directly, without having to write the effective potential, by use of the standard methods of perturbation theory.

In this paper we test the method of stationary variance [10, 11] that has been shown to be viable in simple Abelian gauge theories like QED[30]. According, the

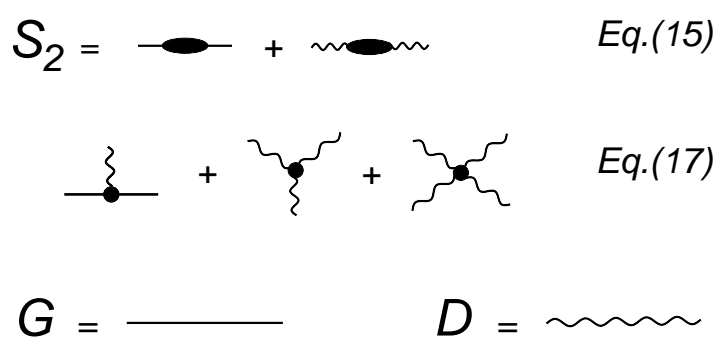

Figure 1: The two-point vertices in the interaction $S_{2}$ of Eq. (15) are shown in the first line. The ghost vertex and the three- and four-gluon vertices of Eqs. (17) are shown in the second line. In the last line the ghost (straight line) and gluon (wavy line) trial propagators are displayed. 


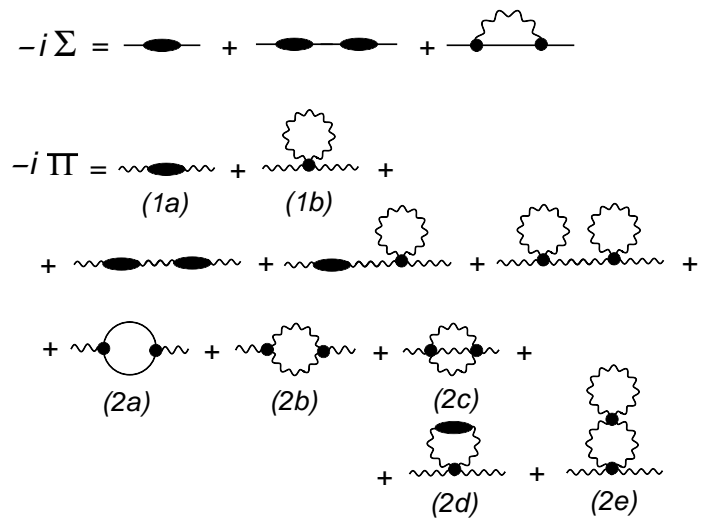

Figure 2: First and second order two-point graphs contributing to the ghost self energy and the gluon polarization. Second order terms include non-irreducible graphs.

self-energy graphs are required up to second order, since the equation for stationary variance can be derived by the general connection that has been proven in Ref. [29]

$$
\begin{gathered}
\frac{\delta V_{n}}{\delta D_{\mu \nu}^{a b}(p)}=\frac{i}{2}\left(\Pi_{n}^{\nu \mu, b a}(p)-\Pi_{n-1}^{\nu \mu, b a}(p)\right), \\
\frac{\delta V_{n}}{\delta G_{a b}(p)}=-i\left(\Sigma_{n}^{b a}(p)-\Sigma_{n-1}^{b a}(p)\right),
\end{gathered}
$$

where the nth-order gluon polarization function $\Pi_{n}^{\mu \nu, a b}$ and the nth-order ghost self-energy $\Sigma_{n}^{a b}$ are the sum of all nth-order connected two-point graphs without tadpoles, while $V_{n}$ is the nth-order term of the effective potential. First and second order two-point graphs are shown in Fig.2.

For $n=2$ the second order term $V_{2}$ is the variance and its stationary conditions follow as

$$
\begin{aligned}
\Pi_{2}^{\nu \mu, a b}(p) & =\Pi_{1}^{\nu \mu, a b}(p) \\
\Sigma_{2}^{b a}(p) & =\Sigma_{1}^{b a}(p) .
\end{aligned}
$$

These are the general stationary conditions that we will use in this paper.

The choice of Feynman gauge, $\xi=1$, simplifies the calculation once we take

$$
D_{\mu \nu}^{a b}(p)=\delta_{a b} \eta_{\mu \nu} D(p)=\delta_{a b} \eta_{\mu \nu} \frac{f(p)}{-p^{2}}
$$

where $D(p)$ is an unknown trial function and $f(p)$ is a trial gluon dressing function. That choice is perfectly legitimate, but is equivalent to a variation of the trial propagator inside a more limited class of functions. More generally, if we take

$$
D_{\mu \nu}^{a b}(p)=\delta_{a b} t_{\mu \nu}(p) D(p)
$$

where $t_{\mu \nu}$ is a given gauge dependent tensor, the functional derivative can be written as

$$
\frac{\delta}{\delta D(p)}=\sum_{a b, \mu \nu} \int \frac{\mathrm{d}^{4} k}{(2 \pi)^{4}} \frac{\delta D_{\mu \nu}^{a b}(k)}{\delta D(p)} \frac{\delta}{\delta D_{\mu \nu}^{a b}(k)}
$$

and by Eq. (22) we can write

$$
\frac{\delta}{\delta D(p)}=\sum_{a b, \mu \nu} \delta_{a b} t_{\mu \nu}(p) \frac{\delta}{\delta D_{\mu \nu}^{a b}(p)} .
$$

Thus, if we define the summed quantity

$$
\Pi_{n}(p)=\frac{1}{4\left(N^{2}-1\right)} \sum_{a b, \mu \nu} \delta_{a b} t_{\mu \nu}(p) \Pi_{n}^{\mu \nu, a b}(p)
$$

and insert it in Eq.(18), the functional derivative of $V_{2}$ with respect to $D(p)$ yields the simple stationary equation

$$
\Pi_{2}(p)=\Pi_{1}(p)
$$

which replaces the first of Eqs. (20).

In this paper we will limit to the special case of Feynman gauge and take $t_{\mu \nu}=\eta_{\mu \nu}$ in the calculation. An interesting alternative would be the choice of the Landau gauge, $\xi=0$. In any case, color symmetry ensures that we can always take

$$
G_{a b}(p)=\delta_{a b} G(p)=\delta_{a b} \frac{\chi(p)}{p^{2}}
$$

where $\chi(p)$ is a trial ghost dressing function.

Despite their simple shape, the stationary equations contain all the one- and two-loop graphs displayed in Fig.2, and are a set of coupled non-linear integral equations for the trial functions $D, G$. It is not obvious that a solution does exist, but we will show in the next sections that a solution can be found by a numerical integration.

\section{STATIONARY EQUATIONS}

Before attempting a numerical solution of the stationary equations it is useful to write them in more detail, in terms of proper (1PI) and reducible graphs.

The first order terms can be easily written as a sum of the first-order graphs of Fig.2. We have a single tree graph $\Sigma_{1}$ for the ghost self energy (the first graph in Fig.2) and making use of the explicit form of the vertices in the interaction Eq. (14) we can write

$$
-i \Sigma_{1}^{a b}(p)=i \delta_{a b}\left[p^{2}-G^{-1}(p)\right]
$$

The first-order gluon polarization has a tree graph $\Pi_{1 a}$ and a one-loop term $\Pi_{1 b}$ as shown in the second line of Fig.2

$$
\begin{aligned}
& -i \Pi_{1 a}^{\mu \nu, a b}=i \delta_{a b} \eta^{\mu \nu}\left[D_{0}^{-1}-D^{-1}\right] \\
& -i \Pi_{1 b}^{\mu \nu, a b}=i \delta_{a b} \eta^{\mu \nu}\left(3 N g^{2}\right) I_{0}^{(1)}
\end{aligned}
$$

where, according to Eq.(16), $D_{0}^{-1}(p)=-p^{2}$ and the integrals $I_{n}^{(m)}$ are constant terms that in the Euclidean 
formalism can be written in terms of the dressing function as

$$
I_{n}^{(m)}=\int \frac{\mathrm{d}^{4} k_{E}}{(2 \pi)^{4}} \frac{\left[f\left(k_{E}\right)\right]^{m}}{\left(k_{E}^{2}\right)^{n+1}} .
$$

We assume that these integrals are made finite by a regulating scheme as discussed below. Details on the calculation of this and all the other loop graphs of Fig.2 are given in the appendix. The sum in Eq. (25) is trivial and yields

$$
\Pi_{1}=D^{-1}-\Delta^{-1}
$$

where we have defined a renormalized zeroth-order massive propagator $\Delta$ as

$$
\Delta(p)=\frac{1}{-p^{2}+M^{2}}
$$

and the mass term $M^{2}$ is defined by the gap equation

$$
M^{2}=3 N g^{2} I_{0}^{(1)}=3 N g^{2} \int \frac{\mathrm{d}^{4} k_{E}}{(2 \pi)^{4}} D\left(k_{E}\right) .
$$

We observe that, as a first order approximation, the gap equations of the GEP are equivalent[29] to the selfconsistency conditions $\Pi_{1}=0$ and $\Sigma_{1}=0$ that yield the simple decoupled analytical solution

$$
\begin{aligned}
& G(p)=\frac{1}{p^{2}} \\
& D(p)=\Delta(p)
\end{aligned}
$$

with free propagators for ghosts and massive gluons.

The second order terms require the sum of all the other graphs displayed in Fig.2. It is useful to introduce a proper polarization function $\Pi_{2}^{\star}$ and a proper self energy $\Sigma_{2}^{\star}$, that are defined as the sum of second-order 1PI graphs, i.e. the last graph of the first line and the last five graphs at the bottom respectively in Fig.2. Assuming a sum over all indices according to Eq.(25) and recalling the diagonal matrix structure of first order terms in Eqs.(28), (29) we can write the second order functions as

$$
\begin{aligned}
& \Pi_{2}=\Pi_{2}^{\star}+\left(\Pi_{1}\right)^{2} D \\
& \Sigma_{2}=\Sigma_{2}^{\star}+\left(\Sigma_{1}\right)^{2} G
\end{aligned}
$$

where the second term on the right hand side is the sum of the reducible graphs, and we are using the obvious notation $\Sigma_{n}^{a b}=\delta_{a b} \Sigma_{n}$. Inserting Eq.(31) and Eq.(28), the stationary conditions of Eq.(20) now take the following form

$$
\begin{aligned}
G(p) & =\frac{1}{p^{2}}-\frac{\Sigma_{2}^{\star}(p)}{p^{4}} \\
D(p) & =\Delta(p)-[\Delta(p)]^{2} \Pi_{2}^{\star}(p) .
\end{aligned}
$$

Thus we only need to consider the 1PI graphs contributing to the proper second-order functions. This pair of coupled non-linear integral equations is well suited for an iterative numerical solution. They can be written in terms of the dressing functions, and switching to the Euclidean formalism we can write them as

$$
\begin{aligned}
& \chi\left(p_{E}\right)=\left[1+\frac{1}{p_{E}^{2}} \Sigma_{2}^{\star}\left(p_{E}\right)\right] \\
& f\left(p_{E}\right)=\frac{p_{E}^{2}}{p_{E}^{2}+M^{2}}\left[1-\frac{\Pi_{2}^{\star}\left(p_{E}\right)}{p_{E}^{2}+M^{2}}\right] .
\end{aligned}
$$

Of course an iterative solution of these equations requires a numerical evaluation of the one- and two-loop graphs contributing to the second-order proper functions $\Pi_{2}^{\star}, \Sigma_{2}^{\star}$ that we need at each step as functionals of the unknown trial dressing functions $f, \chi$. The details on the numerical evaluation of the graphs are reported in Appendix A.

\section{COMPARISON WITH OTHER VARIATIONAL METHODS}

Before going to the detail of the numerical solution, we would like to compare the formal results of the previous sections with other variational methods that have been proposed.

The GEP is probably the simplest variational approach and it gives a dynamical mass generation for the gluon as shown in Eqs.(34). Moreover the same result cannot be obtained by perturbation theory and is a genuine nonperturbative result. In fact, the mass term $M^{2}$ comes out from the one-loop tadpole graph $\Pi_{1 b}$ that vanishes in dimensional regularization when evaluated by inserting the zeroth-order gluon propagator: perturbation theory cannot predict a finite mass at any order. On the other hand, the first order stationary conditions of the GEP require a self-consistent solution with a mass that is evaluated by the gap equation, Eq.(33), and can be written as

$$
M^{2}=3 N g^{2} \int_{\Lambda} \frac{\mathrm{d}^{4} k_{E}}{(2 \pi)^{4}} \frac{1}{k_{E}^{2}+M^{2}}
$$

where $\int_{\Lambda}$ means that the integral has been regularized by a suitable cutoff $\Lambda$, like in non-perturbative lattice calculations. While the integral does not vanish even in dimensional regularization, the simple cutoff regularization seems to be more suited for a direct comparison with lattice calculations.

Among the shorthands of the GEP we mention the constant mass, which does not decrease at large moments, and mainly the known difficulties for dealing with fermions [22, 28, 29] like quarks and even ghosts. In fact, according to Eqs.(34), the ghosts are decoupled and do not play any role in the GEP.

Recently, a technique has been developed for including untrivial effects of the fermions in the GEP [22, 26], and has been used for a non-perturbative study of the Higgstop sector of the standard model $[22]$. The technique, that can be seen as an improvement of RPA, was tested 
in the two-dimensional half-filled Hubbard model, predicting the correct antiferromagnetic limit in the strong coupling limit 26]. It is instructive to see how the technique would improve the GEP, allowing for a correct inclusion of the ghosts. It is based on an exact formal integration of fermions, yielding a pure bosonic effective action. The action is then expanded in powers of the bosonic field, and the expansion is eventually truncated at some order, yielding an approximate action that can be dealt with by a variational method like the GEP. It is quite obvious that truncation spoils the approximation that ceases to be a genuine variational approximation (it is well known that RPA is not a variational approximation). In the present context of a pure $S U(3)$ theory, the exact integration of ghosts gives back the Faddev-Popov determinant

$$
J_{F P}(A)=e^{i S_{e f f}(A)}
$$

that defines the effective action

$$
S_{\text {eff }}(A)=-i \log J_{F P}(A) .
$$

On the other hand $J_{F P}$ is the determinant of the matrix $\mathcal{F}^{a b}(x, y)$ that can be formally written[42]

$$
\mathcal{F}=G_{0}^{-1} \cdot\left[1+G_{0} \cdot B\right]
$$

where the matrix $B^{a b}(x, y)$ is the ghost-gluon vertex in $\mathcal{L}_{g h}$ that can be written as

$$
B^{a b}(x, y)=g f_{a b c} \partial^{\mu} A_{\mu}^{c}(x) \delta^{4}(x-y) .
$$

Then following Ref.[22], the effective action admits the exact expansion

$$
S_{e f f}(A)=S_{e f f}(0)-i \operatorname{Tr} \sum_{n=1}^{\infty} \frac{(-1)^{n+1}}{n}\left[G_{0} \cdot B\right]^{n} .
$$

All these terms must be added to the interaction $S_{I}$ of Eq.(14) and must be regarded as first order vertices to be inserted in the evaluation of the first order polarization $\Pi_{1}$. Then the simple first-order self consistency condition $\Pi_{1}=0$ suffices for determining the gap equation of the improved GEP. Of course, some truncation of the expansion is required in order to have a viable calculation scheme, and the truncation spoils the accuracy of the variational method. In the present context the technique turns out to be equivalent to the expansion proposed by Reinhardt and Feuchter 5 and recently used in the Lagrangian formalism in Ref.[8]. In more detail, the first untrivial term of the expansion is the quadratic one, yielding the correction $\delta S_{I}$

$$
\delta S_{I}=\frac{i}{2} \operatorname{Tr}\left[G_{0} \cdot B \cdot G_{0} \cdot B\right] .
$$

Since $B$ is linear in the field $A$, this interaction term is quadratic, and gives rise to a two-point vertex. By inspection, this composite vertex contains a loop of two ghost propagators connected by two ghost-gluon vertices, and its corresponding first order tree term in the polarization is just the second order 1PI ghost loop $\Pi_{2 a}$ which is displayed in Fig.2. Thus the improved GEP stationary equation $\Pi_{1}=0$ now reads

$$
\Pi_{1}=D^{-1}-\Delta^{-1}+\Pi_{2 a}=0
$$

and gives a massive propagator ${ }^{1}$

$$
D\left(p_{E}\right)=\left[p_{E}^{2}+\Omega^{2}\left(p_{E}\right)\right]^{-1}
$$

with a mass $\Omega(p)$ that depends on $p$ and is given by the modified gap equations

$$
\begin{gathered}
\Omega^{2}\left(p_{E}\right)=M^{2}-\Pi_{2 a}\left(p_{E}\right) \\
M^{2}=3 N g^{2} \int_{\Lambda} \frac{\mathrm{d}^{4} k_{E}}{(2 \pi)^{4}} \frac{1}{k_{E}^{2}+\Omega^{2}\left(k_{E}\right)}
\end{gathered}
$$

where the ghost loop $\Pi_{2 a}$ plays the role of the curvature function of Ref.[8] as shown by Eq.(A8) in the appendix. In that work the approximation is improved by including an infinite class of higher order terms in the expansion Eq.(43). That can be formally done by substituting a dressed ghost propagator $G$ for the bare one $G_{0}$ in the ghost loop, that is equivalent to sum up an infinite series of higher order graphs. As shown in that work the self consistency of the dressed propagator has important effects on the gluon propagator. That seems to be a limit of the simple GEP, while the second order method of stationary variance yields coupled self-consistency equations, Eqs.(37), for the ghost and gluon dressing functions.

A final note on the differences between the GEP and the present method of stationary variance comes from a comparison of the higher-order propagators. We can regard the trial propagators as the starting point of an optimized perturbation theory, and then write higherorder Feynman graphs for the propagators. By Dyson equations, the nth-order propagator $D_{(n)}$ follows from the nth-order proper polarization $\Pi_{(n)}^{\star}$ as ${ }^{2}$

$$
D_{(n)}^{-1}=D^{-1}-\Pi_{(n)}^{\star}
$$

where in general, for $n>1$ the polarization $\Pi_{(n)}^{\star}$ is a matrix, but we omit the indices for brevity. For the GEP,

\footnotetext{
${ }^{1}$ More generally, at first order there is no need to take the special matrix form Eq. (22) and the polarization $\Pi_{2 a}$ can be regarded as a matrix. In that case, provided that $\Pi_{2 a}$ is replaced by $\Pi_{2 a}^{\prime}$ as defined in Eq. A3 , this massive propagator gives just the coefficient of $\eta_{\mu \nu}$ which is the physically relevant part of the propagator. As shown by Eq. A8 in the appendix, $-\Pi_{2 a}^{\prime}$ is formally equal to the curvature function of Ref. [8].

${ }^{2}$ We denote by $X_{(n)}$ the total nth-order value of $X$ while $X_{n}$ is the single nth-order term: $X_{(n)}=\sum_{i=0}^{n} X_{i}$. With the same notation $D \equiv D_{(0)}$ is the zeroth-order approximation.
} 
since $\Pi_{1}=0$ we obtain the self-consistency condition $D_{(1)}=D$. This property is lost at higher orders: in the present scheme of the stationary variance the total second-order proper polarization is

$$
\Pi_{(2)}^{\star}=\Pi_{1}+\Pi_{2}^{\star}=D^{-1}-\Delta^{-1}+\Pi_{2}^{\star}
$$

and the second order propagator reads

$$
D_{(2)}^{-1}=\Delta^{-1}-\Pi_{2}^{\star}=p_{E}^{2}+M^{2}-\Pi_{2}^{\star}\left(p_{E}\right)
$$

A comparison with Eqs.(46), (47) shows that the second order gluon propagator extends the improved GEP [22, 26] or the curvature approximation of Ref.[8] by substituting the whole second order proper self-energy $\Pi_{2}^{\star}$ for the single ghost loop $\Pi_{2 a}$ (the curvature of Ref. [8]). By itself that does not imply a better approximation, but we expect a richer description at least, and an improvement of gauge invariance as the graphs $\Pi_{2 a}$ and $\Pi_{2 b}$ are now summed together as they should.

\section{REGULARIZATION AND NUMERICAL SOLUTIONS}

The method of stationary variance provides a set of coupled non-linear integral equations for the dressing functions. However, there is no proof that the stationary conditions Eqs. (37) have any solution at all. Actually, for any choice of the bare coupling $g$, Eqs. (37) can be iterated and show a fast convergence towards a stable solution. The existence of a stable and physically reasonable solution for the method of stationary variance is one of the main achievements of the present paper, since the method can be developed further as a non-perturbative tool for the study of QCD. An analytical proof of existence was only given before under some special constraints and for the simpler case of an Abelian gauge theory [30].

For a numerical solution of the coupled set of stationary conditions, Eqs. (37), we first need to regularize all the diverging integrals that are reported in detail in the appendix. Dimensional regularization does not seem to be the best choice for a non-perturbative variational approach because of the unknown form of the trial propagators that would require a spectral representation as in Ref.[30]. Moreover, we cannot rely on a perturbative renormalization, order by order, but rather we should consider a non-perturbative multiplicative renormalization scheme. Since the variational method is not gauge invariant, the regulator can even break gauge symmetry, as we expect that gauge invariance should be recovered in physical observables only approximately in the present approximation. In that respect the gauge parameter $\xi$ could even be regarded as a further trial parameter of the variational method, to be determined by its stationary value according to the method of minimal sensitivity [43].

The simple choice of an energy cutoff in the Euclidean space $p_{E}^{2}<\Lambda^{2}$ has the merit of giving physical results that are directly comparable with lattice simulations where a finite lattice acts just like an energy cutoff.
Moreover, lattice simulations are the most natural benchmark for any variational calculation in the low energy limit. Thus we borrow from lattice simulation the regulating scheme and its physical interpretation in terms of a bare interaction parameter $g=g(\Lambda)$ which is supposed to be dependent on the energy scale $\Lambda$. Renormalization Group (RG) invariance requires that the physical observables are left invariant by a change of scale $\Lambda \rightarrow \Lambda^{\prime}$ that is accompanied by the corresponding change of the bare interaction $g(\Lambda) \rightarrow g\left(\Lambda^{\prime}\right)$. Then, renormalized physical quantities can be defined that do not depend on the cutoff. The theory has only one free parameter, namely the interaction strength $g$ at a given scale $\Lambda$, or the scale $\Lambda$ at a given interaction strength. Once that is fixed, the function $g(\Lambda)$ can be determined by RG invariance. In lattice simulations, the scale $\Lambda$ is determined by a comparison of some physical observables with their actual experimental value. In the present calculation we will limit ourselves to the calculation of the propagators and we will fix the scale by a direct comparison with the available lattice data. It is important to point out that the present regularization scheme does not need the inclusion of any counterterm in the Lagrangian and especially mass counterterms that are forbidden by the gauge invariance of the Lagrangian, but are sometimes included as free parameters.

Since $\Lambda$ is the unique energy scale in the theory, we will basically set $\Lambda=1$ and work in units of the cutoff, at a given bare interaction strength $g$. Thus the choice of $\Lambda$ will be equivalent to fixing the natural energy units. Any numerical solution of the stationary equations would take the form of a generic bare dressing function $f_{B}(x, g)$ where $x=p / \Lambda$ is the Euclidean momentum in units of $\Lambda$ and $g=g(\Lambda)$. Since we only use the Euclidean formalism in this section, we drop the $E$ in the momentum $p_{E}$ and denote by $p$ the Euclidean momentum unless otherwise specified. RG scaling requires that a renormalized function $f_{R}$ can be defined at an arbitrary scale $\mu$ by multiplicative renormalization

$$
f_{R}(p / \mu, \mu)=\frac{f_{B}(p / \Lambda, g)}{Z(g, \mu)}
$$

For instance, as normalization condition we can require that $f_{R}=1$ at $p=\mu$ so that

$$
Z(g, \mu)=f_{B}(\mu / \Lambda, g(\Lambda))
$$

which is a function of $g$ and $\mu$ only, since $\Lambda$ can be regarded as an implicit function of $g$. This kind of renormalization obviously requires that the dressing function shows the scaling property

$$
f_{R}(p / \mu, \mu)=\frac{f_{B}(p / \Lambda, g(\Lambda))}{f_{B}(\mu / \Lambda, g(\Lambda))}=\frac{f_{B}\left(p / \Lambda^{\prime}, g\left(\Lambda^{\prime}\right)\right)}{f_{B}\left(\mu / \Lambda^{\prime}, g\left(\Lambda^{\prime}\right)\right)}
$$

or in other words the renormalized function $f_{R}$ is independent of $\Lambda$ and $g$ and the bare function must satisfy

$$
f_{B}(p / \Lambda, g(\Lambda))=K\left(g, g^{\prime}\right) f_{B}\left(p / \Lambda^{\prime}, g\left(\Lambda^{\prime}\right)\right)
$$




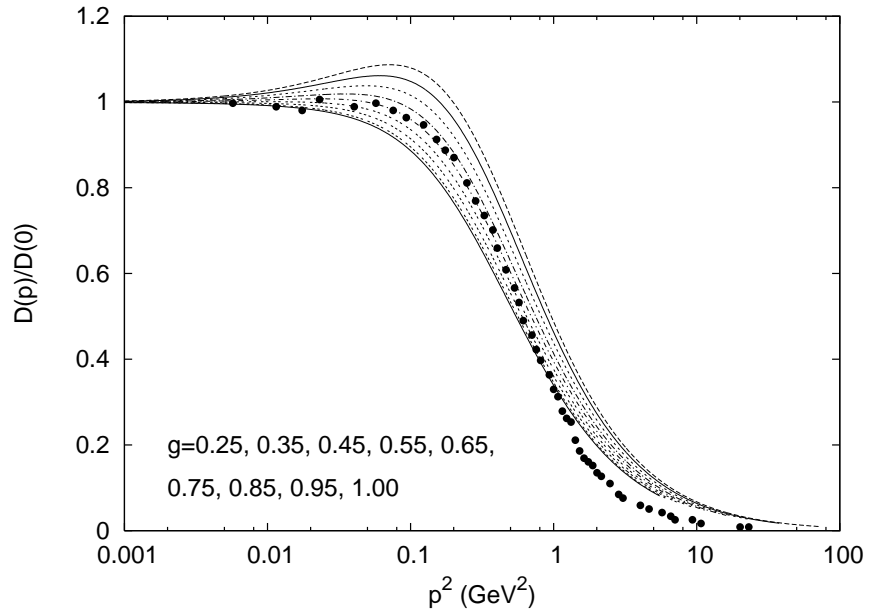

Figure 3: The gluon propagator $D(p) / D(0)$ as a function of the Euclidean momentum for several values of the bare coupling $g=0.25,0.35,0.45,0.55,0.65,0.75,0.85,0.95,1$ (from the top to the bottom). For each bare coupling the energy scale is fixed by taking $M=0.5 \mathrm{GeV}$. The Landau gauge lattice data of Ref.[37] $(g=1.02, \mathrm{~L}=96)$ are reported as filled circles.

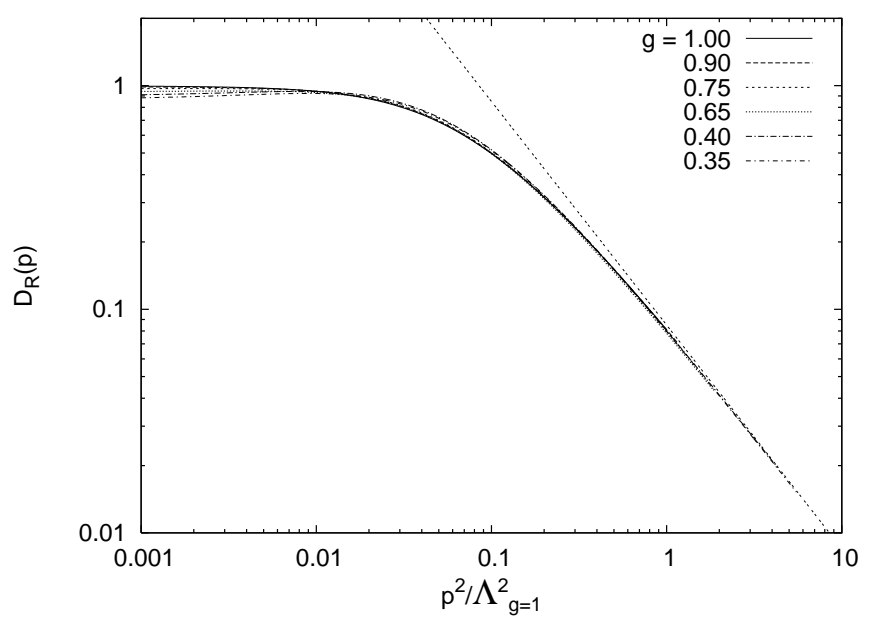

Figure 4: Log-log plot of the renormalized propagator $D_{R}(p)$ as obtained by appropriate scaling of the bare propagator for the bare coupling $g=0.35,0.40,0.65,0.75,0.90,1$. The scale is arbitrary because of scaling: all curves have been scaled in order to fall on top of the $g=1$ bare propagator of Fig.3. Energy is in units of $\Lambda_{g=1}$ so that $g(1)=1$ (for $g=1$ the curve is not rescaled). The dotted straight line is a fit of the asymptotic behavior by $D(p) \approx z / p^{2}$ and $z=0.085$.

where $K\left(g, g^{\prime}\right)$ is a scaling constant that can only depend on $g$ and $g^{\prime}$. This scaling property is evident in a log-log plot of the bare dressing functions since the curves can be put one on top of the other by a simple shift of the axes. Since the approximation and the numerical integration could spoil the scaling properties of the dressing functions, we will consider the scaling as a test for the accuracy of the whole procedure.

As anticipated, in units of the cutoff, the proper po-

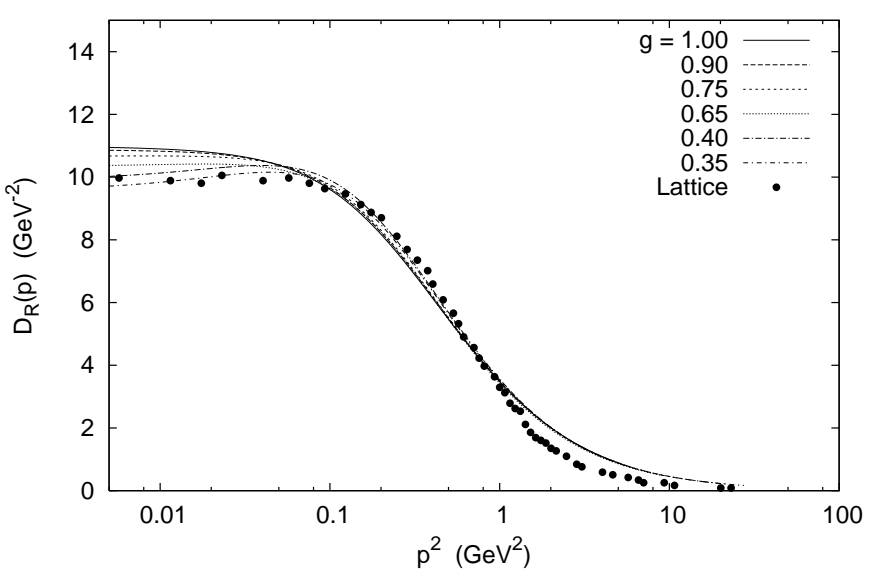

Figure 5: The renormalized propagator $D_{R}(p)$ in physical units for the bare coupling $g=0.35,0.40,0.65,0.75,0.90,1$. Scale factors are the same as in Fig. 4 but the energy scale has been fixed in order to fit the lattice data of Ref. 37] $(g=1.02$, $\mathrm{L}=96$ ) that are displayed as filled circles.

larization and self energy graphs in Fig. 2 are given by finite integrals and can be numerically evaluated as described in detail in the appendix, making use of some initial choice for the trial dressing functions $f$ and $\chi$. Inserting the actual value of the functions $\Pi_{2}^{\star}$ and $\Sigma_{2}^{\star}$ in Eqs.(37), a new pair of dressing functions is obtained and the procedure can be iterated up to self consistency. Convergence is quite fast and special normalization constraints can be imposed on the solutions by Eq.(52) or by other boundary conditions. The gluon propagator is reported in Fig.3 for several values of the bare coupling. For a rough comparison with lattice data, the energy scale is fixed by taking $M=0.5 \mathrm{GeV}$ in physical units. Actually, since the ratio $\tilde{M}=M / \Lambda$ is given by Eq.(33) at any coupling $g$, we are just taking $\Lambda=(0.5 \mathrm{GeV}) /(\tilde{M})$. Lattice data from Ref. 37] are included in the figure, but we must warn that the data of the simulation are obtained in the Landau gauge, while the present calculation is in Feynman gauge. While the propagator is not expected to be gauge invariant, the physical mass should not be too much sensitive to the gauge choice, and we may extract a rough estimate of the energy scale by comparison of the data. Basically, in Fig.3 we are reporting $D(p) / D(0)$ assuming that the mass parameter does not depend on the bare coupling $g$ and is kept fixed at the arbitrary value $M=0.5 \mathrm{GeV}$ that fits the data well enough. This is just a first estimate of the dynamical mass. A more accurate estimate can be obtained by scaling, but depends on the actual definition of mass, which is not obvious as we will see later.

As shown in the log-log plot of Fig.4, by an appropriate change of scale the renormalized propagator $D_{R}(p)$ becomes independent of $g$ and all the curve fall one on top of the other as expected from Eq. (555). Here the single curves are rescaled (just translated in the log-log plot) in 
order to fall on top of the $g=1$ bare propagator. Scaling is rather good with the exception of the far infrared region.

The same curves have been reported in physical units and compared with the lattice data of Ref.[37] in Fig.5. Scale ratios are the same as for Fig.4, but a physical energy scale is fixed in order to give a rough fit of the lattice data. Despite the use of a different gauge, the main features of the lattice propagator seem to be reproduced by the trial function, with a pronounced flat behavior in the infrared. Here we are taking $\Lambda_{g=1}=2.24 \mathrm{GeV}$ and by using the scale factors that come from scaling, the mass parameter $M$ turns out to be slightly dependent on $g$ with a value that goes from $M=0.47 \mathrm{GeV}$ at $g=1$ to $M=0.43 \mathrm{GeV}$ at $g=0.35$. Thus using units of $M$, as we did in Fig.3, does not provides the best scaling.

It is quite obvious that a slightly smaller energy scale in Fig. 5 would give a better agreement in the UV region and a worsening in the IR, with a slight decrease of $M$ in physical units. In other words the renormalized function $D_{R}(p)$ cannot be made to match the lattice data exactly over the whole range of $p$, yielding only approximate estimates of the mass parameter. That could be just a consequence of a different behavior of the propagator in different gauges or it could be a shorthand of the variational approximation. Unfortunately we could not find any recent lattice data in Feynman gauge to compare with.

Besides, it is not obvious that $M$ and $D$ should be the best estimates of the physical mass and propagator. The trial propagator $D$ is just the best zeroth order propagator that optimizes the convergence of the perturbation expansion according to the method of stationary variance. That should give the best effective potential,

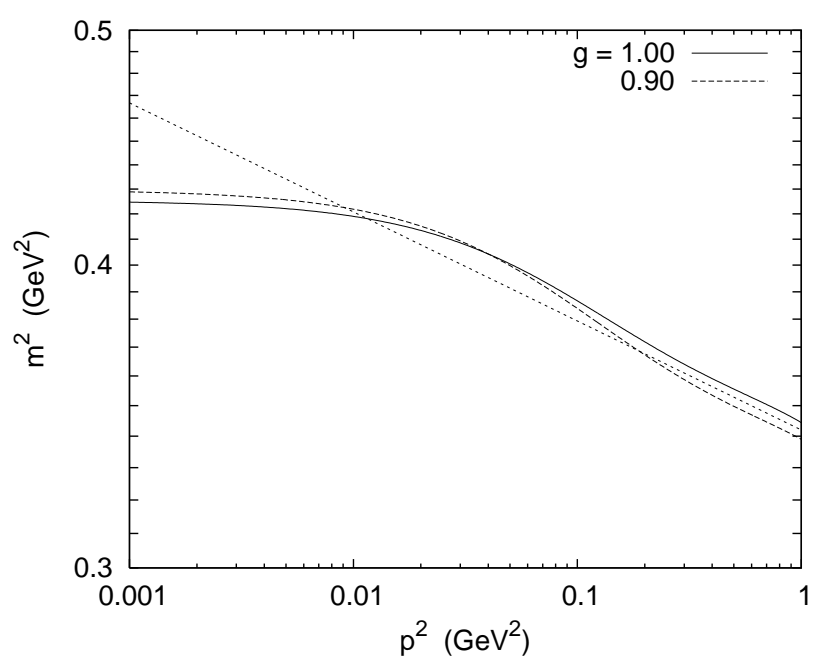

Figure 6: The physical dynamical mass $m^{2}(p)$ is evaluated by Eq.(57) and displayed in a log-log plot for $g=0.9$ and 1 . The leading behavior $m^{2}(p) \approx\left(p^{2}\right)^{-\eta}$ is shown by the dotted straight line for $\eta=0.045$. but the best two-point functions should be extracted as derivatives of the effective potential with respect to the external field[18], and the result could be slightly different. That is probably the best way to approximate the true physical two-point functions in this framework, but it requires the knowledge of the effective action as a functional of the external field, and we leave it as an interesting further development. A more straightforward way to improve on the general behavior of the propagator is provided by the second order approximation of Eq.(51), as we will discuss later in the next section.

We can define a dynamical physical mass $m(p)$ by requiring that

$$
D(p)=\frac{z}{p^{2}+m^{2}(p)}
$$

and that $m$ vanishes in the UV limit. The normalization constant $z$ can be extracted by the asymptotic behavior $D(p) \approx z / p^{2}$ as shown in Fig.4. The mass then follows as

$$
m^{2}(p)=\frac{z}{D(p)}-p^{2}
$$

provided that $p$ is not too large. In the far UV limit the two terms on the right hand side of Eq.(57) are very large and their difference gets very small, so that numerical errors make the result unreliable. We show a log-log plot of $m^{2}(p)$ in Fig.6 for two values of bare coupling $g=1,0.9$. Deviations from the exact scaling get amplified by this procedure, but nevertheless we may extract a power-law behavior $m^{2}(p) \approx\left(p^{2}\right)^{-\eta}$ which is displayed as a straight dotted line in the log-log plot, with $\eta=0.045$. This coefficient seems to be too small when compared to other predictions in Landau gauge [35]. The mass saturates in the IR limit at the value $m(0) \approx 0.65 \mathrm{GeV}$ which is slightly larger than the mass parameter $M \approx 0.47 \mathrm{GeV}$.

\section{SECOND ORDER APPROXIMATION}

The trial propagator $D(p)$, solution of the stationary conditions Eqs.(37), was studied in the previous section as an optimized zeroth order approximation for the physical gluon propagator. However, the dressing functions $f(p), \chi(p)$ can be regarded as just an optimal choice for the zeroth order starting point of the expansion, but they could have no physical relevance. In other words the trial functions $D, G$ don't need to be the physical propagators but they could be regarded as just an infinite set of variational parameters. Of course, if the expansion is optimized, the zeroth order functions must be close to the true physical propagators, and that is the reason why we have traded them as good approximations for the physical propagators until now.

A consistent way to evaluate the physical propagators would require the knowledge of the effective action as a function of the external fields, in order to write the 


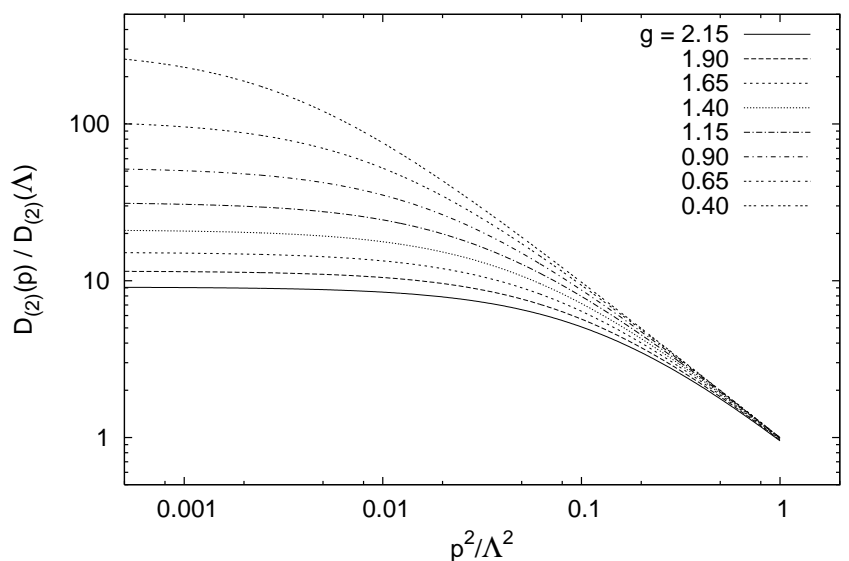

Figure 7: The bare second order propagator $D_{(2)}(p) / D_{(2)}(\Lambda)$ is shown in a log-log plot for $g=0.4,0.65,0.9,1.15,1.4,1.65$, $1.9,2.15$, in units of the cutoff $\Lambda$.

two-point functions as functional derivatives of the effective action[18]. We have left that task to future work. However, it is quite reasonable to think that the actual approximation could also be improved by just adding higher-order Feynman graphs to the propagators $D, G$, using the same Feynman rules as we did before, with an optimized zeroth order action $S_{0}$ and an interaction $S_{I}$ that now are entirely specified by the knowledge of the dressing functions $f, \chi$.

Thus taking $D_{(0)}(p)=D(p)$ and $G_{(0)}(p)=G(p)$ as the free propagators, we can build the higher order functions $D_{(n)}, G_{(n)}$ by Dyson equations like Eq.(49). Dropping the color indices and the $E$ in the Euclidean momentum, the second order function in Eq.(51) can be written in the Euclidean formalism ${ }^{3}$

$$
D_{(2)}^{-1}(p)=\eta_{\mu \nu}\left(p^{2}+M^{2}\right)-\Pi_{2 \mu \nu}^{\star}(p)
$$

and by the same argument

$$
G_{(2)}^{-1}(p)=G^{-1}(p)-\Sigma_{1}(p)-\Sigma_{2}^{\star}(p)
$$

that by Eq.(28) becomes

$$
G_{(2)}^{-1}(p)=\frac{-p^{2}}{\chi_{(2)}(p)}=-p^{2}-\Sigma_{2}^{\star}(p)
$$

where $\chi_{(2)}$ is a second order ghost dressing function that can be written as

$$
\chi_{(2)}(p)=\left[1+\frac{\Sigma_{2}^{\star}(p)}{p^{2}}\right]^{-1}=\frac{1}{\chi(p)} .
$$

\footnotetext{
${ }^{3}$ In this Section, as in the previous one, we drop the $E$ in the momentum $p_{E}$ and denote by $p$ the Euclidean momentum unless otherwise specified.
}

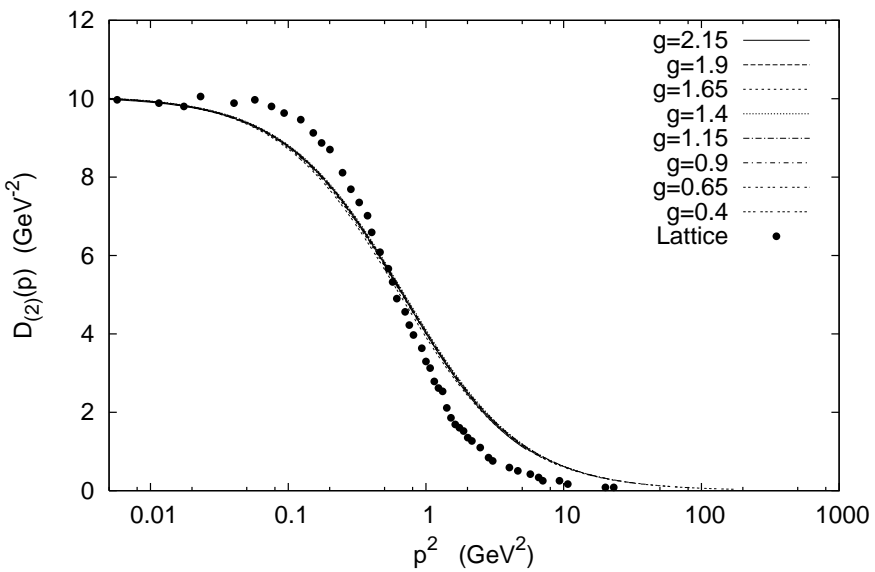

Figure 8: The renormalized second order propagator is shown in physical units for the same couplings of Fig.7. By scaling, all the curves fall one on top of the other. The energy scale is fixed by a rough fit of the Landau-gauge lattice data of Ref.[37] $(g=1.02, \mathrm{~L}=96)$ that are dispayed as filled circles.

It is remarkable that the second order ghost dressing function is just the reciprocal of the zeroth order function $\chi$, thus we do not expect that both of them could give a reasonable approximation for the true dressing function unless $\chi(p)$ is almost constant. That is indeed the case, prompting to a decoupled scenario with ghosts that behave as free particles.

By Lorentz invariance the gluon propagator can be written as

$$
D_{(2)}{ }_{\mu \nu}(p)=\eta_{\mu \nu} D_{(2)}(p)+p_{\mu} p_{\nu} D_{(2)}^{\prime \prime}(p)
$$

where $D^{\prime \prime}$ is the longitudinal component, while $D_{(2)}$ is the physically relevant part we are interested in. ${ }^{4}$ Since $\Pi_{2}^{\star}$ has the same Lorentz structure, we only need the coefficient of $\eta_{\mu \nu}$, denoted by $\Pi_{2}^{\prime}$ in the appendix and evaluated in detail by Eq. A3 for each of the 1PI graphs of Fig.2. The second order gluon propagator $D_{(2)}$ can then be written as

$$
\left[D_{(2)}(p)\right]^{-1}=p^{2}+M^{2}-\Pi_{2}^{\star \prime}(p) .
$$

If we only retain the ghost-loop $\Pi_{2 a}^{\prime}$ and neglect all other polarization graphs, the second-order propagator becomes formally equivalent to that obtained by the curvature approximation of Ref.[8], or by the improved GEP [22, 26] of Eqs.(46), (477). Actually, as shown by Eq. (A8) in the appendix, the function $-\Pi_{2 a}^{\prime}$ is formally equivalent to the curvature of Ref.[8], but the resulting

\footnotetext{
4 Actually, the function $D^{\prime \prime}$ is not relevant in the calculation of any physical observable if the polarization is strictly transversal as required by gauge invariance at any order of perturbation theory. In this calculation the polarization is only approximately transversal.
} 


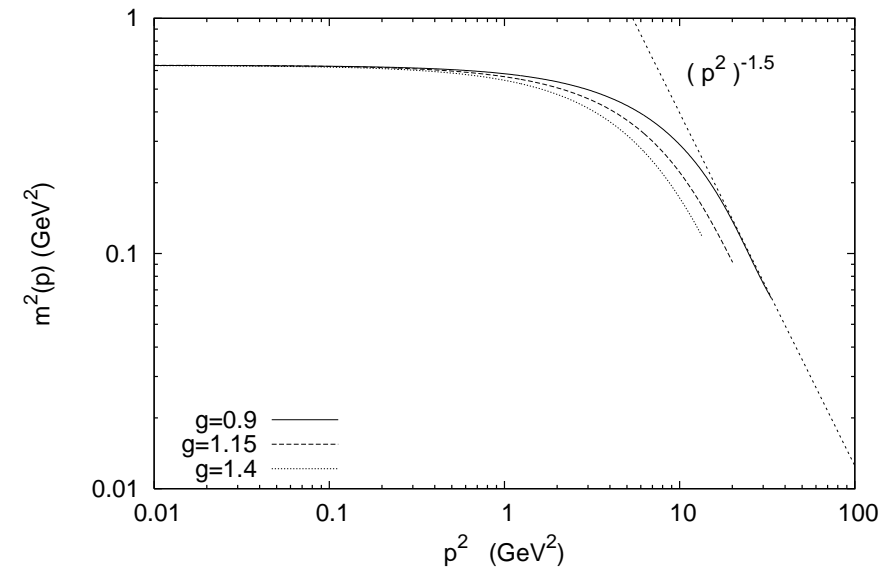

Figure 9: The second-order dynamical mass of Eq. (66) is reported in a log-log plot with the same scaling factors and energy scale of Fig.8, for $g=0.9,1.15$ and 1.4. The straight dotted line shows the power behavior $\sim\left(p^{2}\right)^{-\eta}$ with the exponent $\eta=1.5$.

propagator can be different because of the dressing functions in the loop that have been calculated by different methods. For instance, the ghost dressing function is set to its zeroth order in the GEP, it is given by the coupled stationary conditions of Eqs.(37) in the present method while is given by a closed set of self-consistent DysonSchwinger equations in Ref.[8].

The second order gluon propagator that emerges from Eq. (63) seems to go a step forward by the inclusion of all the 1PI graphs besides the ghost loop. For instance the gluon loop $\Pi_{2 b}^{\prime}$ is now summed together with the ghost loop $\Pi_{2 a}^{\prime}$ as it should be for a correct cancellation of the unphysical degrees of freedom.

The bare second-order propagator is shown in Fig.7 for several values of the bare coupling $g$. As for the trial function $D$, the log-log plot prompts towards the existence of scaling properties. In fact, by a proper scaling, all curves can be put one on top of the other, as displayed in Fig.8 where a physical energy scale has been chosen in order to give a rough fit of the lattice data. While the scaling is now very good, it is painfully obvious that the agreement with the (Landau gauge) lattice data of Ref.[37] is very poor and only a very loose energy scale can be fixed by this method. Once more, we expect that relevant differences may exist between propagators in different gauges and these differences may also depend on the formal definition of the propagator that is not an observable quantity but just an intermediate schemedependent step of the full calculation. Our choice for the scale $\Lambda^{2}$ could become $3 \Lambda^{2}$ or $0.3 \Lambda^{2}$ if we would like to improve the agreement in the IR or in the UV respectively. Any prediction for the dynamical mass also depends on the way the mass is defined, and we have already met different estimates in the study of the trial propagator $D$ in the previous section.
The renormalized second order propagator $D_{(2)}(p)$ in Fig.8 can be fitted quite well by the simple expression

$$
D_{(2)}(p) \approx \frac{Z}{p^{2}+m^{2}}
$$

yielding a physical mass parameter $m \approx 0.8 \mathrm{GeV}$ that is basically independent of $g$. Of course our uncertainty on the energy scale would give something like $0.4 \mathrm{GeV}<$ $m<1.4 \mathrm{GeV}$.

We can introduce a better definition for the dynamical mass if we take

$$
D_{(2)}(p)=\frac{Z}{p^{2}+m^{2}(p)}
$$

where now $m(p)$ is a function which is supposed to decrease as a power, $m^{2} \sim\left(p^{2}\right)^{-\eta}$ for large energies. While Eq.(65) is just a definition for $m$, by Eq.(63) it can be written as

$$
m^{2}(p)=p^{2}(Z-1)+Z\left(M^{2}-\Pi_{2}^{\star \prime}(p)\right) \sim\left(\frac{p^{2}}{p_{0}^{2}}\right)^{-\eta} .
$$

The parameter $Z$ can be tuned in order to get a powerlaw behavior that would appear as a linear curve in a $\log$-log plot. For any bare coupling we find $Z \approx 1$, as we expected by the knowledge of the exact asymptotic limit. For instance, in the case of $g=0.9$ the asymptotic behavior of $m(p)$ is fitted by $Z=0.9978$. The exponent turns out to be $\eta=1.5$ for any coupling, as shown in Fig.9 where the function $m^{2}(p)$ is reported for some different values of the bare coupling, with the same scaling factors and energy scale of Fig.8. Scale dependent values of $\eta$, oscillating in the range $1.08<\eta<1.26$, have been reported in Landau gauge by Ref.[35]. While this kind of plot enhances minor deviations from the exact scaling, we find the same high-energy power-law behavior for different couplings, with a dynamical mass $m(p)$ that saturates at $m(0) \approx 0.8 \mathrm{GeV}$.

Since we have seen that $Z \approx 1$, the second-order propagator can be written as $D_{(2)}^{-1}=p^{2}+m^{2}(p)$ with a dynamical mass that takes the simple form

$$
m^{2}(p)=M^{2}-\Pi_{2}^{\star \prime}(p),
$$

suggesting that the full polarization matrix could be written as the sum of a constant shift plus a transverse polarization term

$$
\Pi_{2 \mu \nu}^{\star}(p)=-\left(\delta m^{2}\right) \eta_{\mu \nu}-\pi(p)\left(\eta_{\mu \nu}-\frac{p_{\mu} p_{\nu}}{p^{2}}\right)
$$

where $\delta m^{2}=m^{2}(0)-M^{2}$ is a constant second-order mass shift and $\pi(p)$, the coefficient of the transverse part, must vanish in the low energy limit, $\pi(0)=0$, yielding

$$
m^{2}(p)=m^{2}(0)+\pi(p) .
$$

While Eq. (68) is suggestive, it is not obvious in any way that it should hold since it requires that

$$
-\pi(p)=\left[\Pi_{2}^{\star \prime}(p)-\Pi_{2}^{\star \prime}(0)\right]=-\Pi_{2}^{\star \prime \prime}(p)
$$


having denoted by $\Pi_{2}^{\star \prime}$ and $\Pi_{2}^{\star \prime \prime}$ the coefficents of $\eta_{\mu \nu}$ and $p_{\mu} p_{\nu} / p^{2}$ respectively, in the proper second-order polarization function, with the notation of Eq. (A1). These functions are defined in detail in the appendix in Eqs. (A2), (A3). The transversality (up to a constant) of the polarization function is what we would expect by gauge invariance in presence of a dynamical mass. It is not obvious that it should hold in the present approximate scheme, and it is generally achieved by a correct cancellation of the unphysical degrees of freedom by the ghost loops. Thus, it is remarkable that Eqs. (68), (70) hold, albeit approximately, in the present variational calculation.

It is instructive to go in detail and look at the behavior of the single terms of the polarization. As expected, Eq.(70) is not satisfied by the single graphs of Fig.2. For instance, in Fig.10, for the ghost loop $\Pi_{2 a}{ }^{\mu \nu}$ and the gluon loop $\Pi_{2 b}{ }^{\mu \nu}$, the functions $\Pi^{\prime}$ and $-\Pi^{\prime \prime}$ are displayed at a bare coupling $g=1.2$, in units of the cutoff. The functions $\Pi^{\prime}$ have been shifted by a constant in order to have $\Pi^{\prime}(0)=0$ for all the single terms. We observe that the transversality condition of Eq.(70) would require that the shifted functions should satisfy $\Pi^{\prime} \approx-\Pi^{\prime \prime}$ at least. That is not the case in Fig.10.

Now let us look at the functions $\Pi^{\prime}, \Pi^{\prime \prime}$ for the total polarization function, including all the 1PI second-order graphs of Fig.2. The function $\Pi^{\prime}$, now shifted by the constant term $\delta m^{2}$, is shown in Fig.11 and compared to $\Pi^{\prime \prime}$. Albeit approximately, $\Pi^{\prime} \approx-\Pi^{\prime \prime}$ when all graphs are added together. Actually, the ghost and gluon loops almost satisfy the transversality condition when added together, but the accuracy improves when all the graphs are summed. Since the loops are evaluated in terms of the self-consistent dressing functions, the transversality condition Eq. (70) seems to be an important test for the

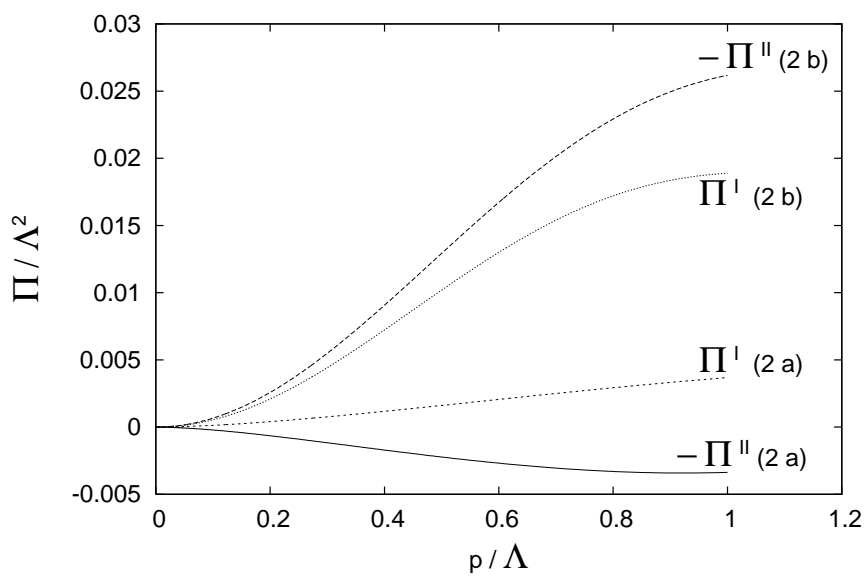

Figure 10: The polarization functions $\Pi^{\prime},-\Pi^{\prime \prime}$ for the ghostloop graph $(2 a)$ and the gluon-loop graph (2b) are displayed for a bare coupling $g=1.2$, in units of the cutoff. The functions $\Pi^{\prime}$ have been shifted by a constant in order to have $\Pi^{\prime}(0)=0$. The transversality condition of Eq.(70), requiring that $\Pi^{\prime} \approx-\Pi^{\prime \prime}$, is not satisfied by the single terms.

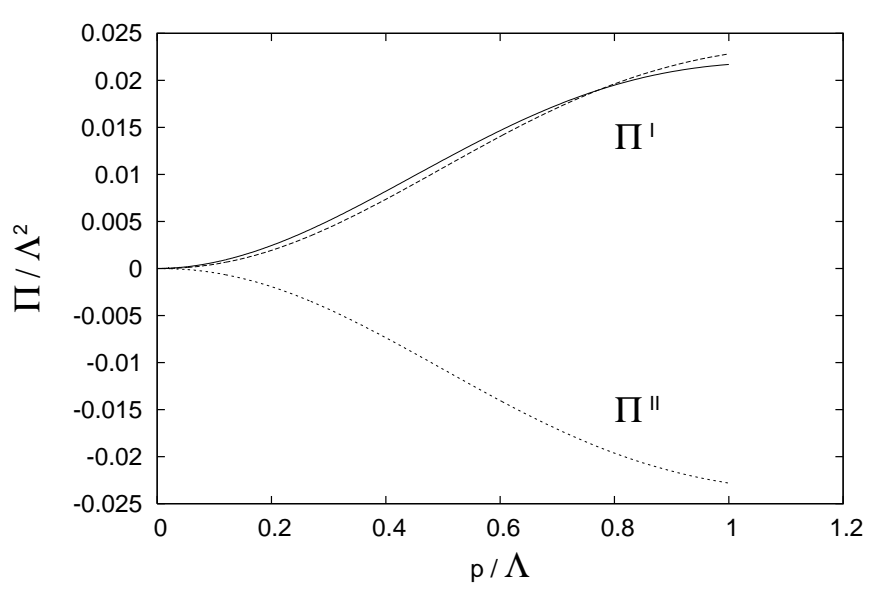

Figure 11: Functions $\Pi^{\prime}, \Pi^{\prime \prime}$ for the total polarization function, including all the 1PI second-order graphs of Fig.2. The function $\Pi^{\prime}$ is shifted by the constant term $\delta m^{2}$ in order to have $\Pi^{\prime}(0)=0$. The opposite of $\Pi^{\prime \prime}$, namely $-\Pi^{\prime \prime}$, is also shown as a dotted line for a direct comparison. We observe that $\Pi^{\prime} \approx-\Pi^{\prime \prime}$, and the transversality condition Eq. (70) is approximately satisfied when all graphs are added together.

overall reliability of the calculation.

Finally, a note on the ghost dressing functions is in order. Since ghosts are not physical, their properties are expected to be even more sensitive to a change of gauge. While the gluon mass can have a physical meaning, no physical observable can be clearly related to ghosts. In fact ghosts can also disappear in a convenient gauge. In Feynman gauge, even a finite ghost propagator, with a vanishing dressing function in the IR limit, would be still consistent with a finite gluon propagator as shown by a very recent study 41]. Thus we have no clear way to say how accurate our ghost dressing function is and any comparison with Landau gauge lattice data seems to be

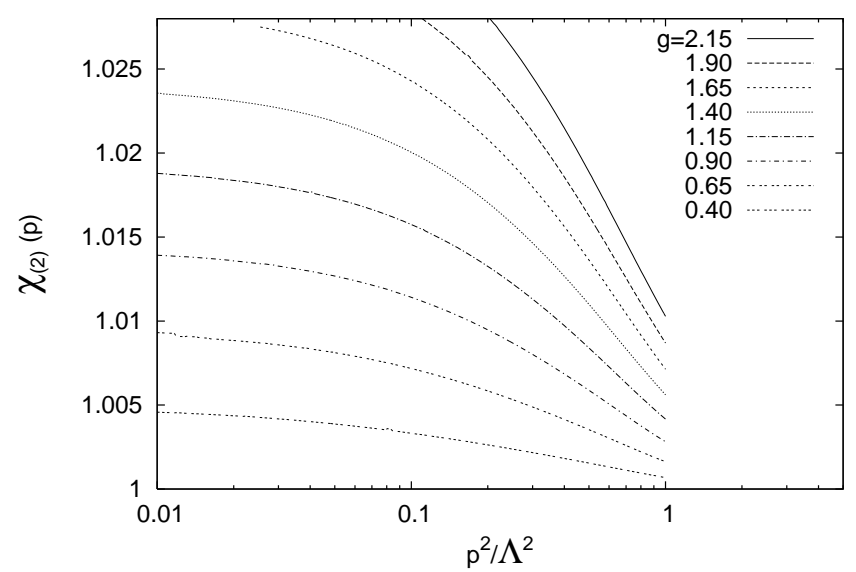

Figure 12: The bare second-order ghost dressing function $\chi_{(2)}$ for several values of the bare coupling $g$, in units of the cutoff. 
even more questionable than it already was for gluons. We can just say that the ghost dressing function seems to play well its role of canceling the unphysical degrees of freedom, as discussed before, and that should suffice. Both $\chi$ and $\chi_{(2)}$ are rather flat functions, with a decoupling scenario of almost free ghosts and an IR finite gluon propagator. We do not see any evidence of a vanishing of the dressing function in the IR limit. In order to give a full picture, the bare second-order ghost dressing function $\chi_{(2)}$ is displayed in Fig.12 for several values of the bare coupling $g$. Despite the scale, the curves are very flat as compared to Landau gauge lattice data.

\section{DISCUSSION}

One of the major achievements of the present paper is the proof that a physically consistent solution does exist for the coupled set of non-linear integral equations that arise from the condition of stationary variance. Since pure Yang-Mills theory already contains fermions (the ghosts), inclusion of quarks in the formalism is straightforward, and would open the way to a broader study of QCD by the same method.

Feynman gauge is also interesting by itself, because the IR behavior of the theory is basically unexplored yet in that gauge. The general picture that emerges from the calculation confirms the decoupling scenario, with a finite ghost dressing function, a finite gluon propagator in the IR limit, and a dynamical mass that decreases as a power in the UV limit. Any quantitative estimate of the gluon mass requires that an accurate energy scale should be fixed first: without any lattice data available in Feynman gauge, we can only give a roughly approximate guess of the scale. By comparison with Landau-gauge lattice data, a saturating value $m(0) \approx 0.5-0.8 \mathrm{GeV}$ is found, depending on the precise definition of mass and energy scale. That estimate is in agreement with other predictions in Feynman gauge 44]. From a qualitative point of view, we cannot confirm the prediction of a finite ghost propagator that has been recently argued[41]. The ghost propagator diverges in the IR limit, and the ghost behaves like a free zero-mass particle.

It is an open question if the second order approximation of Section VI really improves the gluon propagator. By a comparison with Landau-gauge lattice data the optimal trial function $D(p)$ reproduces the flat IR behavior quite well in Fig.5, while the agreement worsen for the second order function $D_{(2)}$ in Fig.8. Of course, we cannot take too seriously a comparison between different gauges, but in principle the second order approximation could even spoil the variational result. If we trust the comparison of the optimal trial function $D(p)$ with the Landau gauge data, than a bit more accurate estimate of the scale can be done, and the dynamical mass saturates at a smaller value $m(0) \approx 0.65 \mathrm{GeV}$ as shown in Fig.6. The functions $D(p)$ and $D_{(2)}(p)$ can be regarded as different approximations, and for that reason we studied in detail the features of both of them in Section V and VI respectively. Needless to say, we need some lattice data in Feynman gauge for answering the open question.

The method can be improved in many way. We did not bother about gauge invariance in this first approach, but the properties of the polarization function, namely the correct cancellations of the unphysical degrees of freedom by the ghosts, show that the constraints of gauge invariance can be satisfied, at least approximately, by the variational solution. While some attempts could be made for enforcing gauge invariance 3, 31], a physically motivated choice for the gauge would probably improve the approximation. Landau gauge would be a good candidate, as it would enforce the transversality in the polarization function from the beginning. An other interesting further development would come from the extension of the formalism to the general case of a finite external background field. For a scalar theory that kind of approach allows a consistent definition of approximate vertex functions by the functional derivative of the effective action. For the GEP these functions can be shown to be the sum of an infinite set of bubble graphs [18]. A similar approach would give a more consistent approximation for the gluon propagator in the present variational framework. Eventually, the inclusion of quarks would lead to a direct comparison with the low energy phenomenology of QCD.

\section{Appendix A: Explicit evaluation of the graphs and numerical details}

We give explicit integral representations of the 1PI graphs that are displayed in Fig.2. The graphs are evaluated by standard Feynman rules, with the trial functions $i G$ and $i D$ associated to any internal ghost and gluon line respectively, and the standard QCD vertices that can be read from the Lagrangian terms in Eqs.(17).

On general grounds, by Lorentz invariance and color symmetry, any generic term contributing to the gluon polarization function can be written as

$$
\Pi_{\mu \nu}^{a b}(p)=\delta_{a b}\left[\eta_{\mu \nu} \Pi^{\prime}(p)+\frac{p_{\mu} p_{\nu}}{p^{2}} \Pi^{\prime \prime}(p)\right] .
$$

The functions $\Pi^{\prime}$ and $\Pi^{\prime \prime}$ can be extracted by saturating the indices with different choices for the tensor $t_{\mu \nu}$ in Eq.(25). Taking $t_{\mu \nu}=\eta_{\mu \nu}$ Eq.(25) yields

$$
\Pi(p)=\frac{1}{32} \sum_{a b, \mu \nu} \delta_{a b} \eta^{\mu \nu} \Pi_{\mu \nu}^{a b}(p)=\Pi^{\prime}(p)+\frac{1}{4} \Pi^{\prime \prime}(p)
$$

while taking $t_{\mu \nu}(p)=\eta_{\mu \nu}-p_{\mu} p_{\nu} / p^{2}$

$$
\Pi^{\prime}(p)=\frac{1}{24} \sum_{a b, \mu \nu} \delta_{a b}\left(\eta_{\mu \nu}-\frac{p_{\mu} p_{\nu}}{p^{2}}\right) \Pi_{\mu \nu}^{a b}(p) .
$$

The function $\Pi^{\prime \prime}$ follows as $\Pi^{\prime \prime}=4\left(\Pi-\Pi^{\prime}\right)$. 
The function $\Pi$ is the one required for the stationary equations and must be inserted in Eqs. (37) for the evaluation of the self consistent solution. The function $\Pi^{\prime}$ has been used for the evaluation of the physically relevant part of the propagator at higher orders in Eq. 63.

The numerical integration has been performed by successive one-dimensional integrations by the standard Simpson method in the Euclidean space and with an energy cutoff $p_{E}^{2}<\Lambda^{2}$. Four-dimensional integrals of simple functions of $k_{E}^{2}$ are reduced to simple one-dimensional integrals before numerical integration, according to

$$
\int_{\Lambda} \frac{\mathrm{d}^{4} k_{E}}{(2 \pi)^{4}} A\left(k_{E}^{2}\right)=\frac{1}{8 \pi^{2}} \int_{0}^{\Lambda} A\left(k^{2}\right) k^{3} \mathrm{~d} k
$$

Four-dimensional integrals of functions of the two variables $\left(k_{E} \cdot p_{E}\right)$ and $k_{E}^{2}$ are reduced to two-dimensional integrals according to

$$
\begin{aligned}
\int_{\Lambda} \frac{\mathrm{d}^{4} k_{E}}{(2 \pi)^{4}} A\left[\left(k_{E} \cdot p_{E}\right), k_{E}^{2}\right]= \\
=\int_{0}^{\Lambda} \frac{y^{2} \mathrm{~d} y}{4 \pi^{3}} \int_{-\sqrt{\Lambda^{2}-y^{2}}}^{\sqrt{\Lambda^{2}-y^{2}}} A\left[\left(x p_{E}\right),\left(x^{2}+y^{2}\right)\right] \mathrm{d} x .
\end{aligned}
$$

\section{Graph (2a)}

The ghost loop $\Pi_{2 a}$ can be written as

$$
\Pi_{(2 a)}^{c d}(p)=-i g^{2} f_{a b c} f_{b a d} \int \frac{\mathrm{d}^{4} k}{(2 \pi)^{4}} i G(p+k) i G(k)\left(p_{\mu}+k_{\mu}\right) k_{\nu}=-\delta_{c d} N g^{2} \int \frac{i \mathrm{~d}^{4} k}{(2 \pi)^{4}} \frac{\left(p_{\mu}+k_{\mu}\right) k_{\nu}}{(p+k)^{2} k^{2}} \chi(p+k) \chi(k)
$$

where a minus sign has been inserted because of the fermion loop. We can saturate the indices as shown in Eqs. (A2), and write in the Euclidean space

$$
\begin{gathered}
\Pi_{2 a}\left(p_{E}\right)=-\frac{N g^{2}}{4} \int \frac{\mathrm{d}^{4} k_{E}}{(2 \pi)^{4}} \frac{\chi\left(p_{E}+k_{E}\right) \chi\left(k_{E}\right)}{\left(p_{E}+k_{E}\right)^{2} k_{E}^{2}}\left(p_{E} \cdot k_{E}+k_{E}^{2}\right) \\
\Pi_{2 a}^{\prime}\left(p_{E}\right)=-\frac{N g^{2}}{3} \int \frac{\mathrm{d}^{4} k_{E}}{(2 \pi)^{4}} \frac{\chi\left(p_{E}+k_{E}\right) \chi\left(k_{E}\right)}{\left(p_{E}+k_{E}\right)^{2}}\left(1-\frac{\left(p_{E} \cdot k_{E}\right)^{2}}{p_{E}^{2} k_{E}^{2}}\right) .
\end{gathered}
$$

We observe that the function $-\Pi_{2 a}^{\prime}$ is the curvature function of Ref.[8] as expected by Eq. (47) or Eq. (51). Actually $\Pi_{2 a}^{\prime}$ is the correct function that must be inserted in Eqs.(47), (51) in order to extract the physically relevant part of the gluon propagator.

\section{Graph (2b)}

The gluon loop $\Pi_{2 b}$ can be written as

$$
\begin{array}{r}
-i \Pi_{(2 b)}^{a d}(p)=\frac{g^{2}}{2} f_{a b c} f_{d b c} \int \frac{\mathrm{d}^{4} k}{(2 \pi)^{4}} i D(p+k) i D(k)\left\{\left(2 p_{\tau}+k_{\tau}\right) \eta_{\mu \rho}-\left(2 k_{\mu}+p_{\mu}\right) \eta_{\rho \tau}+\left(k_{\rho}-p_{\rho}\right) \eta_{\mu \tau}\right\} \times \\
\times\left\{\left(p_{\nu}+2 k_{\nu}\right) \eta^{\rho \tau}-\left(k^{\tau}+2 p^{\tau}\right) \eta_{\nu}^{\rho}+\left(p^{\rho}-k^{\rho}\right) \eta_{\nu}^{\tau}\right\}
\end{array}
$$

where a symmetry factor $1 / 2$ has been inserted. By trivial algebra

$$
\Pi_{(2 b)}^{a d}(p)=\delta_{a d} \frac{N g^{2}}{2} \int \frac{i \mathrm{~d}^{4} k}{(2 \pi)^{4}} D(p+k) D(k)\left\{\eta_{\mu \nu}\left(5 p^{2}+2 k^{2}+2 p k\right)+\left(10 k_{\mu} k_{\nu}-2 p_{\mu} p_{\nu}+5 k_{\mu} p_{\nu}+5 p_{\mu} k_{\nu}\right)\right\}
$$

and then taking $t_{\mu \nu}=\eta_{\mu \nu}$, Eq.(25) yields

$$
\Pi_{2 b}\left(p_{E}\right)=\frac{9 N g^{2}}{4} \int \frac{\mathrm{d}^{4} k_{E}}{(2 \pi)^{4}} \frac{f\left(p_{E}+k_{E}\right) f\left(k_{E}\right)}{\left(p_{E}+k_{E}\right)^{2} k_{E}^{2}}\left(p_{E}^{2}+p_{E} \cdot k_{E}+k_{E}^{2}\right),
$$

while Eq.

$$
\Pi_{2 b}^{\prime}\left(p_{E}\right)=\frac{N g^{2}}{2} \int \frac{\mathrm{d}^{4} k_{E}}{(2 \pi)^{4}} \frac{f\left(p_{E}+k_{E}\right) f\left(k_{E}\right)}{\left(p_{E}+k_{E}\right)^{2} k_{E}^{2}}\left[5 p_{E}^{2}+2 p_{E} \cdot k_{E}+2 k_{E}^{2}+\frac{10}{3}\left(k_{E}^{2}-\frac{\left(p_{E} \cdot k_{E}\right)^{2}}{p_{E}^{2}}\right)\right]
$$




\section{Graph (2c)}

Let us denote by $\Gamma_{a b c d}^{\mu \nu \rho \sigma}$ the four-gluon vertex $\mathcal{L}_{4}$ in Eq.(17), that can be written as

$$
\Gamma_{\mu \nu \rho \sigma}^{a b c d}=-i \frac{g^{2}}{4 !}\left[T_{\mu \nu \rho \sigma}^{a b c d}+T_{\mu \rho \sigma \nu}^{a c d b}+T_{\mu \sigma \nu \rho}^{a d b c}\right]
$$

where the matrix structure $T$ is

$$
T_{\mu \nu \rho \sigma}^{a b c d}=f_{e a b} f_{e c d}\left(\eta_{\mu \rho} \eta_{\nu \sigma}-\eta_{\mu \sigma} \eta_{\nu \rho}\right) .
$$

With a symmetry factor $(4 ! 4 ! / 3 !)$, the two-loop term $\Pi_{2 c}$ can be written as

$$
-i \Pi_{(2 c)}{ }_{\mu \tau}^{a f}(p)=\frac{4 ! 4 !}{3 !} \Gamma_{\mu \nu \rho \sigma}^{a b c d} \Gamma_{\tau}^{f b c d}{ }^{\nu \rho \sigma} \int \frac{\mathrm{d}^{4} k}{(2 \pi)^{4}} \int \frac{\mathrm{d}^{4} q}{(2 \pi)^{4}} i D(k) i D(q) i D(k+q+p)
$$

and in terms of the matrix structure $T$

$$
\Pi_{(2 c)}^{a f}{ }_{\mu \tau}(p)=3 \frac{g^{4}}{3 !} T_{\mu \nu \rho \sigma}^{a b c d}\left[T_{\tau}^{f b c d}{ }^{\nu \rho \sigma}+T_{\tau}^{f c d b}{ }^{\rho \sigma \nu}+T_{\tau}^{f d b c}{ }^{\sigma \nu \rho}\right] \int \frac{i \mathrm{~d}^{4} k}{(2 \pi)^{4}} \int \frac{i \mathrm{~d}^{4} q}{(2 \pi)^{4}} D(k) D(q) D(k+q+p)
$$

where the 3 factor in front arises because of the three identical terms in the product $\Gamma \cdot \Gamma$ that only differ for a permutation of dummy indices. The first product is

$$
T_{\mu \nu \rho \sigma}^{a b c d} T_{\tau}^{f b c d}{ }^{\nu \rho \sigma}=2\left(\eta_{\mu \tau} \eta_{\nu}^{\nu}-\eta_{\mu \tau}\right) f_{e a b} f_{e c d} f_{g f b} f_{g c d}=6 \eta_{\mu \tau} N \delta_{e g} f_{e a b} f_{g f b}=6 N^{2} \delta_{a f} \eta_{\mu \tau}
$$

while the other two products are

$$
T_{\mu \nu \rho \sigma}^{a b c d}\left[T_{\tau}^{f c d b}{ }^{\rho \sigma \nu}+T_{\tau}^{f d b c}{ }^{\sigma \nu \rho}\right]=-\left(\eta_{\mu \tau} \eta_{\nu}^{\nu}-\eta_{\mu \tau}\right)\left[f_{e a b} f_{e c d}\left(f_{g f c} f_{g d b}+f_{g f d} f_{g b c}\right)\right]=3 \eta_{\mu \tau} f_{e a b} f_{e c d}\left(f_{g c d} f_{g f b}\right)
$$

having used Jacobi identity in the last equality. The last two lines can be summed together yielding $9 N^{2} \delta_{a f} \eta_{\mu \tau}$ and by Eqs. A2 , (A3) we obtain

$$
\Pi_{2 c}\left(p_{E}\right)=\Pi_{2 c}^{\prime}\left(p_{E}\right)=\frac{9 g^{4} N^{2}}{2} \int \frac{\mathrm{d}^{4} k_{E}}{(2 \pi)^{4}} \int \frac{\mathrm{d}^{4} q_{E}}{(2 \pi)^{4}} \frac{f\left(k_{E}\right) f\left(q_{E}\right) f\left(k_{E}+q_{E}+p_{E}\right)}{k_{E}^{2} q_{E}^{2}\left(k_{E}+q_{E}+p_{E}\right)^{2}} .
$$

Before numerical integration, this eight-dimensional integral is reduced to a four-dimensional one by obvious generalization of Eq. (A5): the internal integration is performed on the two variables $q_{E}^{2}$, $\left[q_{E} \cdot\left(k_{E}+p_{E}\right)\right]$ by Eq. (A5) and the resulting function of $k_{E}$ and $\left(k_{E} \cdot p_{E}\right)$ is integrated again by Eq.(A5).

\section{Constant graphs (1b), (2d) and (2e)}

With a symmetry factor $4 ! / 2$, the one-loop first order polarization $\Pi_{1 b}$ can be written in terms of the four-gluon vertex of Eq. (A13)

$$
-i \Pi_{(1 b)}^{c d}{ }_{\rho \sigma}=\frac{4 !}{2} \Gamma_{\mu \nu \rho \sigma}^{a b c d} \int \frac{\mathrm{d}^{4} k}{(2 \pi)^{4}}\left[i \eta_{\mu \nu} \delta_{a b}\right] D(k)=-i \frac{g^{2}}{2}\left(6 N \delta_{c d} \eta_{\rho \sigma}\right) \int \frac{i \mathrm{~d}^{4} k}{(2 \pi)^{4}} D(k)
$$

yielding the result of Eq.(29) and $\Pi_{1 b}=-M^{2}$ as defined in Eq.(33).

Since the total first order polarization $\Pi_{1}$ is diagonal, we can evaluate the second order terms $\Pi_{2 d}$ and $\Pi_{2 e}$ directly from the first-order one-loop term $\Pi_{1 b}$ by replacing the internal gluon propagator $D$ with the product $D \Pi_{1} D$ in Eq. (A20), yielding

$$
\begin{gathered}
\Pi_{2 d}=3 N g^{2} \int \frac{i \mathrm{~d}^{4} k}{(2 \pi)^{4}} D(k)\left[D^{-1}(k)-D_{0}^{-1}(k)\right] D(k)=3 N g^{2}\left[I_{0}^{(2)}-I_{0}^{(1)}\right] \\
\Pi_{2 e}=3 N g^{2} \int \frac{i \mathrm{~d}^{4} k}{(2 \pi)^{4}} D(k)\left[-M^{2}\right] D(k)=3 N g^{2} M^{2} I_{1}^{(2)}=M^{4} \frac{I_{1}^{(2)}}{I_{0}^{(1)}} .
\end{gathered}
$$




\section{One-loop ghost self-energy}

The second-order proper self energy graph (third graph in Fig.2) can be written as

$$
\Sigma_{2}^{\star a d}(p)=g^{2} f_{c b a} f_{c d b} \int \frac{i \mathrm{~d}^{4} k}{(2 \pi)^{4}}(-k \cdot p) i D(p+k) i G(k)
$$

and switching to the Euclidean space

$$
\Sigma_{2}^{\star a d}\left(p_{E}\right)=\delta_{a d}\left[N g^{2} \int \frac{\mathrm{d}^{4} k_{E}}{(2 \pi)^{4}} \frac{f\left(k_{E}+p_{E}\right) \chi\left(k_{E}\right)}{\left(k_{E}+p_{E}\right)^{2} k_{E}^{2}}\left(k_{E} \cdot p_{E}\right)\right]
$$

[1] C. S. Fischer, A. Maas, and J. M. Pawlowski, Annals Phys. 324, 2408 (2009), arXiv:0810.1987, and references herein

[2] C. Lerche and L. von Smekal, Phys.Rev. D65, 125006 (2002), arXiv:hep-ph/0202194, and references herein

[3] I.L. Kogan and A. Kovner, Phys. Rev. D 52, 3719 (1995)

[4] C. Feuchter and H. Reinhardt, Phys. Rev. D 70, 105021 (2004).

[5] H. Reinhardt and C. Feuchter, Phys.Rev. D 71, 105002, (2005).

[6] D. Epple, H. Reinhardt, W. Schleifenbaum, A.P. Szczepaniak, Phys. Rev. D77, 085007,(2008), arXiv:0712.3694

[7] Hugo Reinhardt, Davide R. Campagnari, Adam P. Szczepaniak, Phys. Rev. D84, 045006 (2011), arXiv:1107.3389

[8] M. Quandt, H. Reinhardt, J. Heffner, Phys. Rev. D 89, 065037 (2014)

[9] A. P. Szczepaniak, Phys.Rev. D 69, 074031 (2004).

[10] F. Siringo and L. Marotta, Eur. Phys. J. C 44, 293 (2005).

[11] F. Siringo, Mod. Phys. Lett. A, Vol. 29, No. 5 (2014) 1450026, arXiv:1308.4037

[12] L.I. Schiff, Phys. Rev. 130, 458 (1963).

[13] G. Rosen, Phys. Rev. 172, 1632 (1968).

[14] T. Barnes and G. I. Ghandour, Phys. Rev. D 22 , 924 (1980).

[15] P.M. Stevenson, Phys. Rev. D 32, 1389 (1985).

[16] F. Siringo, Phys. Rev. D 62, 116009 (2000).

[17] F. Siringo, Europhys. Lett. 59, 820 (2002).

[18] F. Siringo and L. Marotta, Int. J. Mod. Phys. A25, 5865 (2010), arXiv:0901.2418v2.

[19] R. Ibañez-Meier, I. Stancu, P.M. Stevenson, Z. Phys. C 70, 307 (1996).

[20] F. Siringo, L. Marotta, Phys. Rev. D 78, 016003 (2008).

[21] F. Siringo and L. Marotta, Phys. Rev. D 74, 115001 (2006).

[22] F. Siringo, Phys. Rev. D 86, 076016 (2012), arXiv: 1208.3592v2.

[23] M. Camarda, G.G.N. Angilella, R. Pucci, F. Siringo, Eur. Phys. J. B 33, 273 (2003).
[24] L. Marotta, M. Camarda, G.G.N. Angilella and F. Siringo, Phys. Rev. B 73, 104517 (2006).

[25] C. K. Kim, A, Rakhimow, Jae Hyung Hee, Eur. Phys. Jour. B 39, 301 (2004).

[26] L. Marotta and F. Siringo, Mod. Phys. Lett. B, 26, 1250130 (2012), arXiv:0806.4569 v3.

[27] I. Stancu and P. M. Stevenson, Phys. Rev. D 42, 2710 (1990).

[28] I. Stancu, Phys. Rev. D 43, 1283 (1991).

[29] F. Siringo, Phys. Rev. D 88, 056020 (2013), arXiv:1308.1836

[30] F. Siringo, Phys. Rev. D 89, 025005 (2014), arXiv:1308.2913

[31] A. C. Aguilar, J. Papavassiliou, Phys. Rev. D81, 034003 (2010), arXiv:0910.4142

[32] A. C. Aguilar, D. Binosi, J. Papavassiliou, Phys. Rev. D78, 025010 (2008), arXiv:0802.1870

[33] A. C. Aguilar, D. Binosi, J. Papavassiliou, J. Rodriguez-Quintero, Phys. Rev. D80, 085018 (2009), arXiv:0906.2633

[34] A. C. Aguilar, D. Ibanez, J. Papavassiliou, Phys. Rev. D 87, 114020 (2013), arXiv:1303.3609

[35] A. C. Aguilar, D. Binosi, J. Papavassiliou, Phys. Rev. D 89, 085032 (2014), arXiv:1401.3631

[36] A. C. Aguilar, D. Binosi, D. Ibanez, J. Papavassiliou, arXiv:1405.3506

[37] I.L. Bogolubsky, E.M. Ilgenfritz, M. Muller-Preussker, A. Sternbeckc, Phys. Lett. B 676, 69 (2009), arXiv:0901.0736 3

[38] A. Cucchieri and T. Mendes, Phys. Rev. D 71, 051902(R) (2005)

[39] A. Cucchieri and T. Mendes, arXiv:0710.0412 v 1

[40] J. M. Cornwall, Phys. Rev. D 26, 1453 (1982)

[41] A. C. Aguilar, J. Papavassiliou, Phys.Rev. D 77, 125022 (2008), arXiv:0712.0780v1

[42] S. Weinberg, The quantum theory of fields, Vol.II, Cambridge University Press (1996).

[43] P. M. Stevenson, Phys. Rev. D 23, 2916 (1981).

[44] A collection of gluon mass estimates can be found in Table I of Ref. [35]. 